\title{
Effect of Prescription of Traditional Sports Regimen on Depression of College Students
}

\author{
Yu Ling ${ }^{1}$, Xia Jun-mei ${ }^{1 *}$ \\ ${ }^{1}$ Dept.P.E. of Central South University, Changsha 410083
}

\begin{abstract}
To explore the effect of prescription of traditional sports regimen with different amounts of exercise on improving depression and sleep quality of college students. Depression Screening Scale (PHQ-9) and Zung's Self-rating Depression Scale (SDS) were used to screen students with mild depression, who were randomly divided into control group, experimental group 1 (moderate exercise intensity, 30min each time, 5 times a week,) and experimental group 2 (moderate exercise intensity, 60min each time, 5 times a week). The exercise prescription intervention with different exercise amount was performed for 16 weeks. Compared with before exercise, the total scores of SDS and PSQI of the two experimental groups decreased significantly after 8 weeks of intervention; after 16 weeks of intervention, the total scores of SDS and PSQI of the experimental groups were obviously different $(\mathrm{P}<0.05)$ compared with 8 weeks of intervention; after 8 weeks of training cessation, the total scores of SDS and PSQI of the experimental groups were increased, but however, significant decrease in the total scores still can be seen compared with those before the experiment $(\mathrm{P}<0.05)$. Compared with the control group, after 8 weeks of intervention, 16 weeks of intervention and 8 weeks of training cessation, the total scores of SDS and PSQI of the two experimental groups were significantly reduced and the effect of experimental group 2 on improving PSQI score of college students was significantly better than that of experimental group 1.Traditional sports regimen intervention can improve college students' depression and improve their sleep quality, and it can still maintain the effect after stopping training for 8 weeks; exercise intervention of 60 minutes each time, 5 times a week, has better effect on improving college students' sleep quality than that of 30 minutes each time, 5 times a week.
\end{abstract}

\section{Introduction}

Depression, also known as depressive disorder, is characterized by depression, insomnia, anxiety, lack of interest or pleasure, and even suicide. With the increasingly fierce social competition and the accelerating pace of life, depression presents a high prevalence rate and a high recurrence rate. WHO calls depression the fourth largest disease in the world. Due to their immature physical and mental development, interpersonal communication, pressure of learning and employment, social adaptation and other conditions, college students have become a high incidence group of depression [2]. Research carried out by Tang Hui et al showed that the detection rate of depression among college students in China is $29.3 \%$, which is higher than that of the general population and even more serious than that of social youth of the same age [3]. A large number of studies have shown that regular exercise can alleviate and improve the symptoms of depression and facilitate the recovery of depression. Physical exercise is a natural, active, effective and cheap means to treat depression [4-6]. 'The Outline of the Healthy China 2030 Plan' clearly points out that the active role of national fitness in health promotion, chronic disease prevention and rehabilitation should be given full play. The purpose of this study is to carry out an experimental study on the traditional health care sports prescription for college students with mild depression and to explore the effect of prescription of traditional sports regimen with different amounts of exercise on college students' depression.

\section{Research Object and Method}

\subsection{Research Object}

The survey was conducted among the 2018 freshmen of a comprehensive university. According to the screening criteria of depression, the students with mild depressive symptoms were screened out. After excluding the situation of the physical diseases and dysplasia, those students who met the standards were taken as the research object of this topic. 


\subsection{Research Method}

\subsubsection{Screening College Students with Depression and Experimental Grouping}

The criteria for screening college students with mild depression: The Depression Screening Scale (PHQ-9, The Patient Health Questionnaire-9) score ranges from 5-9 points [4], and The Depression Self-Rating Scale (SDS) standard scores range from 50-62 [5]. According to the results of The Physical Activity Readiness Questionnaire (PAR-Q), combined with mild depression screening conditions, 70 female students (average age 18.7) who volunteered to participate in the experiment were selected and randomly divided into 3 groups: control group (20 people), experiment group 1 (low exercise load group, 25 people) and experimental group 2 (high exercise load group, 25 people).

\subsubsection{Exercise Intervention Program}

The experimental group adopted the exercise intervention scheme with medium intensity, the same exercise content but different exercise time for experimental control (see Table 1). Both the experimental group and the control group participated in college physical education once a week (90min), and the control group did not carry out any intervention. The intensity of exercise was monitored according to the Polar telemetry heart rate monitor watch and The Rating of Perceived Exertion (RPE) finished after exercise.

Table 1 Exercise Intervention Program

\begin{tabular}{|c|c|c|c|}
\hline \multirow{5}{*}{ Content } & & Experiment group 1 & Experiment group 2 \\
\hline & Preparation & Jogging and stretching $(3 \mathrm{~min})$ & Jogging and stretching $(5 \mathrm{~min})$ \\
\hline & Basic part & $\begin{array}{l}\text { Twenty-four Form Simplified Tai Chi; } \\
\text { Twelve methods-Methods of Daoyin }\end{array}$ & $\begin{array}{l}\text { Twenty-four Form Simplified Tai Chi; } \\
\text { Twelve methods-methods of Daoyin }\end{array}$ \\
\hline & & $\begin{array}{c}\text { Yangsheng; Fitness } \\
\text { Qigong · Baduanjin; Qigong · Wuqinxi } \\
(25 \mathrm{~min})\end{array}$ & $\begin{array}{l}\text { Yangsheng; Fitness Qigong · Baduanjin; } \\
\text { Qigong · Wuqinxi;(50min) }\end{array}$ \\
\hline & End part & $\begin{array}{c}\text { Body and mind adjustment exercises } \\
\text { such as pranayama and heart } \\
\text { regulation }(2 \mathrm{~min})\end{array}$ & $\begin{array}{l}\text { Body and mind adjustment exercises such } \\
\text { as pranayama and heart regulation }(5 \mathrm{~min})\end{array}$ \\
\hline Intensity & & $50-70 \% \mathrm{HRmax}$ & 50-70\%HRmax \\
\hline Time & & $30 \mathrm{~min}$ & $60 \mathrm{~min}$ \\
\hline Frequency & & 5 times a week & 5 times a week \\
\hline
\end{tabular}

\subsubsection{Testing Index}

(1) The Self-rating Depression Scale (SDS) [5] is composed of 20 items and 4 factors. The scale mainly requires the subjects to grade 1-4 levels according to their feelings in the last week and evaluates the frequency of symptoms. The higher the score, the more serious the depression. Studies have shown that the scale has good validity and reliability in practical applications [6].

(2) The Pittsburgh Sleep Quality Index(PSQI) [7] consists of 5 other-rated items and 19 self-rated items. The scale is based on the sleep status of the last month and scored by 0-3 levels. The higher the total score, the worse the sleep quality of the subjects.

Standardized instruction languages for the measurement of the scale were strictly used, which were explained by the investigator one by one. No signature was required, and answers were filled out independently.

\subsubsection{Statistics Method}

The SPSS25.0 computer software was used for statistical processing and analysis. The collected data was expressed as $\bar{X} \pm$ S. The paired t-test was used to compare the data before and after the experiment, and the significance level was set to $\alpha=0.05$.

\section{Research Result}

\subsection{Baseline Statistics of Research Subjects}

Before the experiment, there was no significant difference in age, height and weight between the control group, the experimental group 1 and the experimental group 2 (P > $0.05)$. A total of 10 people were removed due to study pressure ( 2 people), unable to keep up with the exercise time (6 people), and incomplete return visit data (2 people) The final effective experimental data is: 23 people in experiment group 1, 18 people in experiment group 2 and 19 people in control group .

Table 2 Baseline Statistics of Research Subjects

\begin{tabular}{ccccc}
\hline & Control & Experiment & Experiment & Totol \\
& Group & Group 1 & Group 2 & \\
Rejected Samples & 1 & 2 & 7 & 10 \\
Sample Size(n) & 19 & 23 & 18 & 60 \\
\hline
\end{tabular}




\begin{tabular}{ccccc}
\hline Age(years old) & $18.53 \pm 0.77$ & $18.48 \pm 0.85$ & $18.39 \pm 0.85$ & $18.47 \pm 0.81$ \\
Height $(\mathrm{cm})$ & $161.0 \pm 3.1$ & $160.9 \pm 4.5$ & $161.1 \pm 5.5$ & $161.0 \pm 4.4$ \\
weight $(\mathrm{kg})$ & $55.6 \pm 18$ & $51.6 \pm 13.0$ & $57.9 \pm 12.1$ & $54.7 \pm 14.5$ \\
BMI & $21.4 \pm 6.8$ & $20.0 \pm 5.0$ & $22.3 \pm 4.4$ & $21.1 \pm 5.5$ \\
\hline
\end{tabular}

\subsection{Effect of Prescription of Traditional Sports Regimen on SDS total score of College Students}

As is shown in Table 3, before the intervention of the traditional sports regimen prescription, the total SDS scores of the experimental group 1,2 and the control group were not significantly different, suggesting that the total SDS scores of the control group and the experimental groups were at the same level. Compared with preexercise, after 8 weeks of exercise prescription intervention, the total SDS score of the experimental group of college students decreased, but there was no significant difference; after 16 weeks of exercise prescription intervention, the total SDS score of the experimental groups of college students decreased significantly, with a significance at the 0.05 level $(\mathrm{P}<0.05)$. After stopping the intervention for 8 weeks, the total SDS score of the experimental group improved, but compared with the score before the experiment, there was still a significant obvious difference $(\mathrm{P}<0.05)$.

Compared with the control group, after 8 weeks of intervention, 16 weeks of intervention and 8 weeks of cessation, the total SDS scores of experimental group 1 and experimental group 2 were distinctly lower, and there was significant difference between intervention for 16 weeks and stopping intervention for 8 weeks $(\mathrm{P}<0.05)$, but there was no significant difference between experimental group 1 and experimental group 2.

Table3 Comparison of SDS Total Score of Each Group Before and After the Experiment $\left(\bar{X}_{ \pm S}\right)$

\begin{tabular}{ccccc}
\hline & Before & After 8 weeks & After 16 weeks & After 8 weeks \\
& Experiment & intervention & intervention & cessation \\
Control Group & $57.47 \pm 2.63$ & $57.74 \pm 2.79$ & $57.84 \pm 2.75$ & $57.95 \pm 2.32$ \\
Experimental Group 1 & $57.61 \pm 2.52$ & $56.52 \pm 2.64$ & $54.96 \pm 3.51^{* \# \#}$ & $56.04 \pm 2.33^{*} \#$ \\
Experimental Group 2 & $56.67 \pm 2.22$ & $55.94 \pm 1.76 \#$ & $54.22 \pm 3.27 * * \# \#$ & $54.89 \pm 1.97 * \# \#$ \\
\hline
\end{tabular}

Note: In the experimental group, before and after the experiment *: $\mathrm{P}<0.05 ;{ }^{* *}: \mathrm{P}<0.01$

Before and after the experiment, the experimental group compared with the control group \#: $\mathrm{P}<0.05$; \#\#:P $<0.01$

\subsection{Effect of Prescription of Traditional Sports Regimen on Sleep Quality of Depression College Students}

Before the intervention of traditional sports regimen, there was no significant difference in the total score of PSQI between the experimental group 1,2 and the control group (see Table 4) and the sleep quality of the control group and the experimental group was at the same level. Compared with the scores before exercise, after 8 weeks of intervention, the total score of PSQI of experimental group 1 and group 2 decreased significantly, with obvious difference $(\mathrm{P}<0.05)$; after 16 weeks of intervention, the total score of PSQI of experimental group 1 and group 2 continued to decline, with a very significant difference $(\mathrm{P}$ $<0.01$ ); after stopping intervention for 8 weeks, the total score of PSQI of experimental group 1 and group 2 increased, but there was still significant difference compared with that before the experiment $(\mathrm{P}<0.05)$.

Compared with the control group, the total score of PSQI of experimental group 1 and group 2 was significantly decreased after 8 weeks of intervention, 16 weeks of intervention and 8 weeks of stopping intervention. After 8 weeks of intervention and 16 weeks of intervention, the sleep quality of experimental group 1 was significantly different $(\mathrm{P}<0.05)$, and the sleep quality of experimental group 2 had a very significant difference $(\mathrm{P}<0.01)$.

Table4 Comparison of PSQI total score of each group before and after the experiment $(\bar{X} \pm \mathrm{S})$

\begin{tabular}{ccccc}
\hline & Before & After 8 weeks & After 16 weeks & After 8 weeks \\
& Experiment & intervention & intervention & cessation \\
Control Group & $7.05 \pm 1.27$ & $6.89 \pm 0.88$ & $6.79 \pm 0.98$ & $6.84 \pm 1.07$ \\
Experimental Group 1 & $7.13 \pm 1.22$ & $6.26 \pm 0.92^{*} \#$ & $6.09 \pm 1.12^{* * \#}$ & $6.30 \pm 1.06^{*}$ \\
Experimental Group 2 & $7.17 \pm 1.10$ & $6.11 \pm 0.76^{* * \# \#}$ & $5.89 \pm 1.02^{* * \# \#}$ & $6.28 \pm 0.89^{*}$ \\
\hline
\end{tabular}

Note: In the experimental group, before and after the experiment *: $\mathrm{P}<0.05 ; \quad * *: \mathrm{P}<0.01$

Before and after the experiment, the experimental group compared with the control group \#: $\mathrm{P}<0.05$; \#\#:P $<0.01$

\subsection{Analyses of the Rationality of Exercise Prescription}

According to the results of heart rate monitoring in the experimental process (Fig. 1), the average heart rate of college students in experimental group 1 and group 2 is between 120 and 140 beats $\cdot \mathrm{min}^{-1}$, which indicates that the exercise intensity of implementing traditional sports regimen is in medium intensity, further verifying the rationality of exercise prescription. 


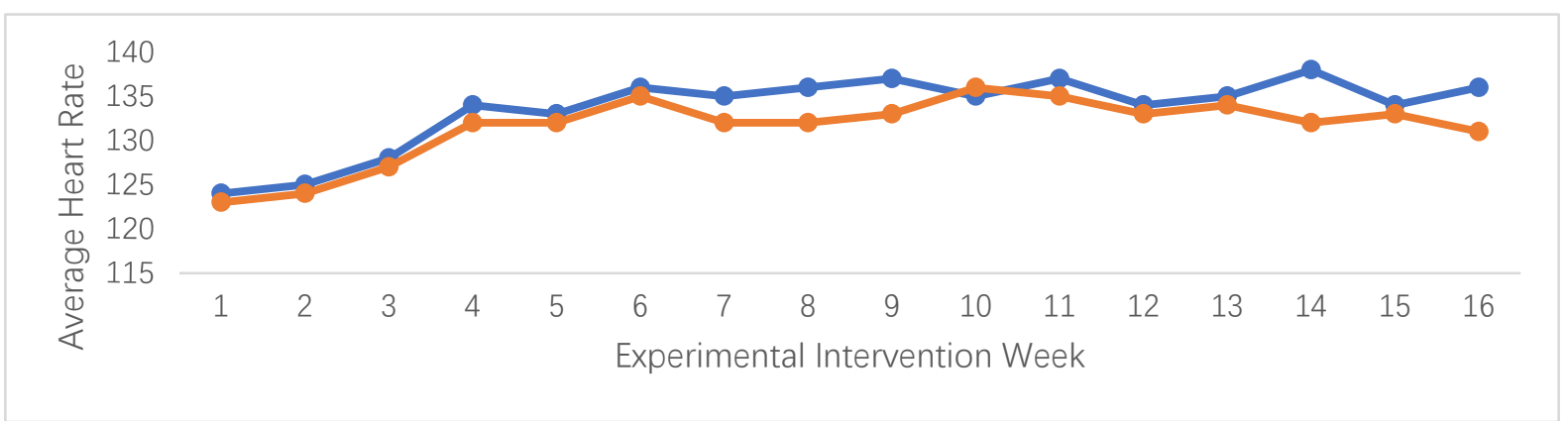

Figure 1 Average curve of average heart rate in experimental exercise intervention

\section{Discussion}

\subsection{Effect of Traditional Sports Regimen Prescription on Relieving Depression of College Students}

This research lasted for 24 weeks. According to the final data collected, 2 people in the experimental group 1 and 7 people in the experimental group 2 withdrew. It shows that the design of the exercise prescription is different in considering the needs and expectations of students. According to the interview with the subjects, the main reason why the students dropped out of the research was that the students had heavy learning tasks, great learning pressure and too much homework, so they could not adhere to the exercise prescription for 60 minutes after class. The original intention of this research is that the depressed female college students have poor enthusiasm about physical exercise subjectively and have intense learning tasks objectively. If it is difficult for them to adhere to the 60 minute traditional sports health exercise intervention, can we carry out at least 30 minutes of exercise to effectively improve depression.

The results showed that compared with the control group, the experimental group of college students on the basis of the original study and life increasing the traditional sports regimen prescription five times a week, each time 30 minutes or 60 minutes for 16 weeks, significantly reduced the SDS total score of depression college students, and there was no evident difference in the effect of traditional sports regimen with the same exercise intensity and different duration on SDS for 16 weeks. This result supports the experimental results of American psychologists William Morgan [8], which shows that low intensity aerobic exercise three times a week (20-30 minutes) will alleviate depression, and the effect on women is significantly better than that on men; it also supports the research conducted by Yao Chong [5] that the exercise intervention for depression students needs to last at least 9 weeks.

After 8 weeks of training cessation, although the depression level of the experimental group students increased slightly, it was still significantly lower than the baseline level, suggesting that the traditional sports regimen for a certain time can not only effectively improve depression, but also the effect can continue to exist after the exercise. Some scholars have shown that even after one year, these interventions still have a positive impact on depression symptoms. Compared with the conventional aerobic exercise, the traditional exercise prescription adopts a collective exercise mode. First of all, it is easy for the female college students to adhere to [9], which can alleviate individual depression through positive social interaction. Secondly, traditional sports can release, transfer and ease negative emotions [10], calm the mind, so that "shape adjustment" can "rest the mind" . Breathing that is fine, long, even, slow and deep can help to cultivate and train depression students' mentality, temperament and spirit [11]. Also, the idea of yin and yang balance of exercise itself can effectively improve depression. Finally, in the design of exercise prescription, combining the form of exercise with the interests of depression students, and reasonable development and adjustment will produce greater effect.

\subsection{Effect of Traditional Sports Regimen Prescription on Sleep Quality of College Students}

Our investigation shows that the average PSQI score of depression college students is $(7.12 \pm 1.18)$ points, which is significantly higher than the normal score of PSQI of college students $[(5.26 \pm 2.38)$ points $]$, which has significant difference $(\mathrm{t}=12.186, \mathrm{P}<0.001)$. It shows that the sleep quality of college students with depression is obviously poor. Usually depression and sleep disorders occur simultaneously [12]. Poor sleep quality is a major cause of depression. Some literature studies have shown that college students with poor sleep quality are more likely to suffer from depression [13-14]. There is a positive correlation between PSQI score and depression scale score [15].

This study supports the research results of Shen Hejun [16]. Traditional physical exercise can improve the sleep quality of college students, and prescription can improve sleep quality through bidirectional regulation pathway of excitation and inhibition of central nervous biological clock [17]. Traditional sports such as Wuqinxi and Baduanjin are simple, easy to learn and moderate in intensity, which are convenient for college students to adhere to for a long time. Traditional physical exercise can enhance the physical fitness of college students, improve their ability to resist diseases and help improve their sleep quality. With the smoothing and elegant music, depression college students' spirit and body are completely relaxed during group practice, and their movements and breathing 
are combined. Endorphin is produced in the body, which has hypnotic effect and can improve the sleep quality in an all-round way.

Compared with the control group, the total score of PSQI of experimental group 1 and group 2 was significantly different after 8 weeks and 16 weeks of traditional sports prescription intervention. And the effect of experimental group 2 on improving the sleep quality of college students was significantly better than that of experimental group 1, indicating that the amount of exercise was negatively correlated with PSQI, suggesting that the greater the amount of exercise, the faster the improvement of sleep quality, with significant dose effect. It may be related to the increase of plasma melatonin concentration with the increase of exercise duration in traditional sports regimen intervention, which leads to the obvious improvement of sleep quality of college students. This is consistent with the results of Skrinar [18].

\section{Conclusion and Suggestion}

\subsection{Conclusion}

Prescription of traditional sports regimen can improve the mild depression of college students. Adding the traditional sports regimen which lasted for 16 weeks for 30 minutes or 60 minutes five times a week on the basis of the original life can significantly improve the depression level of college students, and it still has a maintenance effect after stopping training for 8 weeks. Traditional sports regimen can improve the sleep quality of college students with mild depression, showing a significant dose-effect relationship. The effect of exercise intervention for 60 minutes, five times a week is better for improving the sleep quality of depression college students.

\subsection{Suggestion}

This study is only a preliminary test on the effect of exercise intervention program for mild depression college students. It is suggested that experimental intervention research should be carried out on students with moderate or severe depression in the future research. We also suggest that the exercise items and exercise amount should be arranged reasonably according to the preference and spare time of the students, and they should be encouraged to actively participate in the exercise intervention. And the intervention expectation and follow-up period is suggested to extend. According to the change trend of college students, the intervention expectation should be extended to one year or two years, and the follow-up period should be extended to six months or even longer, so as to see the long-term intervention effect of traditional sports regimen prescription on depression symptoms of college students.

\section{REFERENCES}

1. Mustaffa S, AzizR, Mahmood M, et a1. Depression and suicidal ideation among university students[J].
Procedia-Social and Behavioral Sciences, 2014,11 6:4205-4208.

2. Shensa A, Cesar C Escobar Viera, Sidani J E. Problematic Social Media Use and Depressive Symptoms Among U. S. Young Adults a Nationally-representative Study[J]. Social Science \& Medicine, 2017(182):150.

3. Tang Hui, Ding Lingling, Song Xiulai, et al. Metaanalysis on the detection rate of depression in Chinese college students from 2002 to $2011[\mathrm{~J}]$. Journal of Jilin University: medical science, 2013, 39(5): 965-969.

4. Zhao Jingbo, Chen Zhuangyou, Liang Shunwei, et al. Mediating effect of emotion regulation efficacy on depression and suicide for medical graduate students[J]. Chinese Journal of Special Education, 2019, 5:72-77

5. Yao Chong, Zhao Shanguang, Mao Zhihong, et al. Effect of physical activity intervention on depression and its metabolic. Mechanism[J]. Journal of Shanxi Normal University (natural science edition), 2019.47 (3): $21-30$

6. Wang Jianzhi, Zheng Hongbao, Ding Jianchang, et al. An analysis on the effect of exercise prescription on depression intervention of medical students[J]. China Higher Medical Education, 2014,4:45-46

7. Nishiyama T, Mizuno T, Kojima M, et al. Criterion validity of the Pittsburgh Sleep Quality Index and Epworth Sleepiness Scale for the diagnosis of sleep disorders[J].Sleep Med, 2014,15(4):422-429.

8. Jun ZMS, Yen ST. Physical activity, gender difference, and depressive symptoms[J]. Health Serv Res, 2015, 50( 5 ):1550-1573.

9. Yu Qian, Li Liguo, Li Enyao. Meta-analysis on the improvement of college students's depression by Taijiquan training[J]. International Journal of Psychiatry,2019.46(3):409-412.

10. Wu Yonghui, Chen Ouying, Lou Yaoyue, et al. The application of Taijiquan and Baduanjin in relieving anxiety and depression in patients with coronary heart disease[J]. Nursing research, 2016,30(32):4050-4052

11. Kong J, Wilson G, Park J, et al. Treating depression with tai chi: state of the art and future perspectives [J]. Front Psychiatry, 2019, 10(4):237.

12. Qu Qunfang, Wu Chuanfang, Tan Yuting, et al. Literature analysis of research on improving depression of Baduanjin[J]. Journal of Traditional Chinese Medicine, 2019,25(3):57-59.

13. Dionisios Bratis, Asimoula Spanopoulou, Silvia Dumitru, et al. Sleep disturbance symptoms and their associations with alexithymia, depression and anxiety.Ann Gen Psychiatry , 2010, 9( Suppl 1) : S163.

14. Yu Xubao, Sun Hongyan, Xiong Kai, et al. Metaanalysis of the relationship between sleep quality and depression in Chinese college students[J]. Z Chinese health statistics,2019,36(3):420-422.

15. Wang Liping. A study on sleep quality, mental health 
and physical exercise of some college students in Chengdu and their correlation[D]. Sichuan Normal University,2008.

16. Shen Hejun, Cao Yanjun, Pei Yue, et al. Influence of different Chinese medicine exercises on sleep quality, depression and anxiety level of college students[J]. Chinese Journal of Traditional Chinese medicine information, 2008,25(2):15-19

17. SCHMUTTE T, DAVIDSON L, O'CONNELL M. Improved sleep, diet, and exercise in adults with serious mental illness : results from a pilot selfmanagement intervention[J]. Psychiatr Q,2017, 88(2): $1-11$.

18. Skrinar, G.S., Bullen, B.A., Reppert, S. M. Melatonin response to exercise training in Women[J].Journal of Pineal Research,1997, 2(1):185 -194 